\title{
The Use of Microprocessor Technology for the Conditional Sampling of Intermittent Ocean Processes
}

\author{
JAMES D. IRISH, Wendell S. BROWN AND ThOMAS L. Howell \\ Ocean Process Analysis Laboratory and Department of Earth Sciences, University of New Hampshire, Durham, NH 03824
}

(Manuscript received 24 August 1983, in final form 9 November 1983)

\section{ABSTRACT}

\begin{abstract}
Geophysical signals are often intermittent, having statistics which vary with time. Optimal sampling of these signals requires a so-called "conditional sampling" scheme, a technique which changes the sampling program to match the time scales of the processes of interest. To optimize the limited tape storage capacity of remote oceanographic instruments, a conditional sampling scheme has been implemented using the computational power of microprocessor-controlled instruments, and several deployments have been made with various configurations of the conditional sampling algorithm. This algorithm monitors short-term changes in the energy of an incoming signal within a designated high-frequency band (by digital filtering techniques) and compares the resulting intensity with the longer term statistics of the signal. If the energy exceeds an intensity defined as critical according to some criteria, then an "event" is declared and the data are recorded at a higher than normal rate for the duration of the event. When the statistics of the expected signals are not well known, and criteria cannot be predetermined with confidence, an "adaptive" technique is required whereby the instrument makes an in situ determination of the critical intensity level for each signal based on the statistics of that signal.

Several deployments of the conditional sampling instruments have been made which demonstrate the operation of the technique. In Massachusetts Bay, a burst of high-frequency internal wave energy was identified and recorded by the adaptive critical algorithm applied to a moored temperature sensor array. On the northern California shelf, salinity was calculated in situ from moored temperature and conductivity sensors, and the resulting salinity time series conditionally sampled to identify salinity events as separate from temperature or pressure events.

Conditional sampling techniques may not be optimum for exploratory work. However, where the processes and expected signals are intermittent and have a specific signature, then the use of a conditional sampling technique can make more efficient use of the limited storage capacity of remote instrumentation.
\end{abstract}

\section{Introduction}

Energy transfer processes in the ocean and atmosphere are intermittent phenomena. High energy events occur only rarely in space and time, but significantly affect the environment. For example, the most severe storms are likely to be associated with a larger momentum and heat flux between the atmosphere and the ocean than are the winds during the rest of the year. A breaking internal wave can mix more heat and salt than other diffusion processes. Tsunami or hurricane-generated waves may contribute more to sediment erosion and coastal damage than the accumulated effects of daily wind waves.

This intermittency makes geophysical data acquisition difficult. Routine sampling at a high fixed rate would provide large quantities of redundant data and require a quantity of storage space not generally available on remotely recording instruments. A fixed low sampling rate would provide inadequate data during the rare occurrences of interesting high-energy, highfrequency events. A program of intermittent rapid sampling superimposed on a standard-low-frequency sampling rate (burst sampling) is better, and works well with quasi-stationary processes such as oceanic swell, where the statistics change slowly over time. However, it is not entirely satisfactory when the statistics of the process are not stationary.

To sample the "events" properly with an instrument of limited storage capacity, a sampling program is needed which employs a high sampling rate contingent on the occurrence of the rare important events-a scheme we refer to as conditional sampling. In effect, this type of sampling program is followed when there is human (intelligent) involvement in monitoring data as it is collected, as in meteorological sampling of severe storms, oceanographic sampling of Gulf Stream rings, etc., wherein the scientist looks at the data in real time and then plans how to sample next.

At the University of New Hampshire (UNH), research efforts on wind-driven shelf circulation have focused on the measurement and interpretation of the oceanic pressure field. Bottom instruments have been developed which employ microprocessor-controlled data loggers to sample both the pressure at the bottom, and temperature and conductivity along a moored ar- 
ray above the bottom instrument. This combination of measurements allows the inference of both barotropic and baroclinic parts of the pressure field. The microprocessor, as part of the recording system, supplies the "intelligence" required to implement a conditional sampling scheme which optimizes the limited storage capacity of the recorder.

In this paper, we describe a conditional sampling scheme which we have implemented on our bottommounted instrumentation. This scheme adjusts the sampling rate to match the energy content in a selected high frequency band, and adapts the definition of an event to the statistics of the past measurements taken by the instrument. We first describe the instrumentation and then discuss a specific conditional sampling scheme. Finally, some illustrative results from several deployments of this new instrumentation are presented and discussed.

\section{Instrumentation}

The UNH microprocessor-controlled recorder uses the INTERSIL IM6100 family of CMOS microprocessor components. Each box in the block diagram shown in Fig. 1 corresponds to a circuit board which plugs into the microprocessor's bus as required to configure the system to the specific requirements of an experiment. The elements of the microprocessor component of the system consist of the CPU, a memory expansion board, Random Access Memory (RAM), Erasable Programmable Read Only Memory (EPROM), a Universal Asynchronous Receiver Transmitter (UART), and a driver to the $1 / O$ bus. Up to three fields of memory (4096 twelve-bit words of memory per field) can be added as required. As configured for our most recent deployment in the Coastal Ocean Dynamics Experiment (CODE), the instrument has three fields of memory as shown in Fig. 1.

The basic operating system, floating-point "math" package, and most of the conditional sampling software are located in Field " 0 ". Field " 1 ", which is entirely. RAM, is used for temporary data storage. Field " 3 " holds the recording software and acoustic subroutines. The system as shown draws $25 \mathrm{ma}$ at $5 \mathrm{~V}$ and has been powered on lithium batteries for up to four months. The digital cassette interfaces directly with the bus and is capable of holding up to $10^{7}$ bits of data on a 300 -foot cassette. In a typical four-month experiment, we allocate half the tape capacity to the recording of routine low-frequency samples from all sensors, and the remainder to conditionally sampled "high-frequency" data.

The timing for the instrument is supplied by a stable $4 \mathrm{MHz}$ quartz crystal clock which controls the basic 15-second sample interval. The clock controls the timing for all counter gates, latches and resets as well as "rebooting" the microprocessor after each sample interval. This rebooting of the system is done to recover from any random bit error which would interrupt the program.

The output from each sensor in the system is a frequency-modulated (FM) signal which is counted on a sensor counter/interface board containing twelve-bit counters and circuitry for three sensors. The FM signal is shaped to a $5 \mathrm{~V}$ square wave, gated and counted under control of the clock. At the end of each sample interval, the twelve least significant bits are transferred into a latch, the counter is reset, and the count is restarted. Since the timing for all sensors is controlled by the same clock, the sensor readings, which are summed over the 15 -second sample interval, are simultaneous. The microprocessor has control over each latch through the sensor control board and transfers each count to the bus at the proper time for storage in RAM.

Pressure is measured with a Paroscientific quartz sensor (see Wearn and Larson., 1982, for an evaluation of these sensors). Temperature is measured with a thermistor-controlled oscillator (Pederson, 1969) and conductivity with an electrode-resistance-controlled oscillator (Pederson and Gregg, 1979) produced by Sea Bird Electronics, Inc. In the instrument configuration presented here, the temperature and conductivity sensor pairs are mounted on a vertical array above the main instrument frame on which the pressure sensor is mounted.

The instrument contains other electronics boards which are not directly involved in recording data. A UART board is connected to an external terminal which is used to initialize the instrument and monitor its operation on deck before deployment and after recovery. This instrument also contains an acoustic diagnostic and alert system, similar to that described by Snodgrass (1968), for sending engineering diagnostic information on the operation of the sensors and recording electronics to a nearby ship. This system permits us to check operation of the instrument after it is in position on the sea floor. As a back-up alternative to the acoustic release used for normal recovery of the instrument, a timer/exploding bolt system is employed. The back-up timer card is programmed to detonate an exploding bolt after a preset number of hours.

With the microprocessor-controlled recorder, the sampling program is controlled by software so that sophisticated conditional sampling schemes can be implemented. After each 15-second sample interval, the digitized count from each sensor is transferred, under software control, from the appropriate latch of the counter board to RAM. The 60 most recent samples from each sensor are temporarily stored, and are the data upon which the conditional sampling scheme operates. The routine 15-minute "low-frequency" sample is formed from a software sum of sixty 15-second samples. Each sum contains the least significant 18 bits of the accumulated frequency count. Each sum is identical to counting the sensor frequency over the entire 15- 


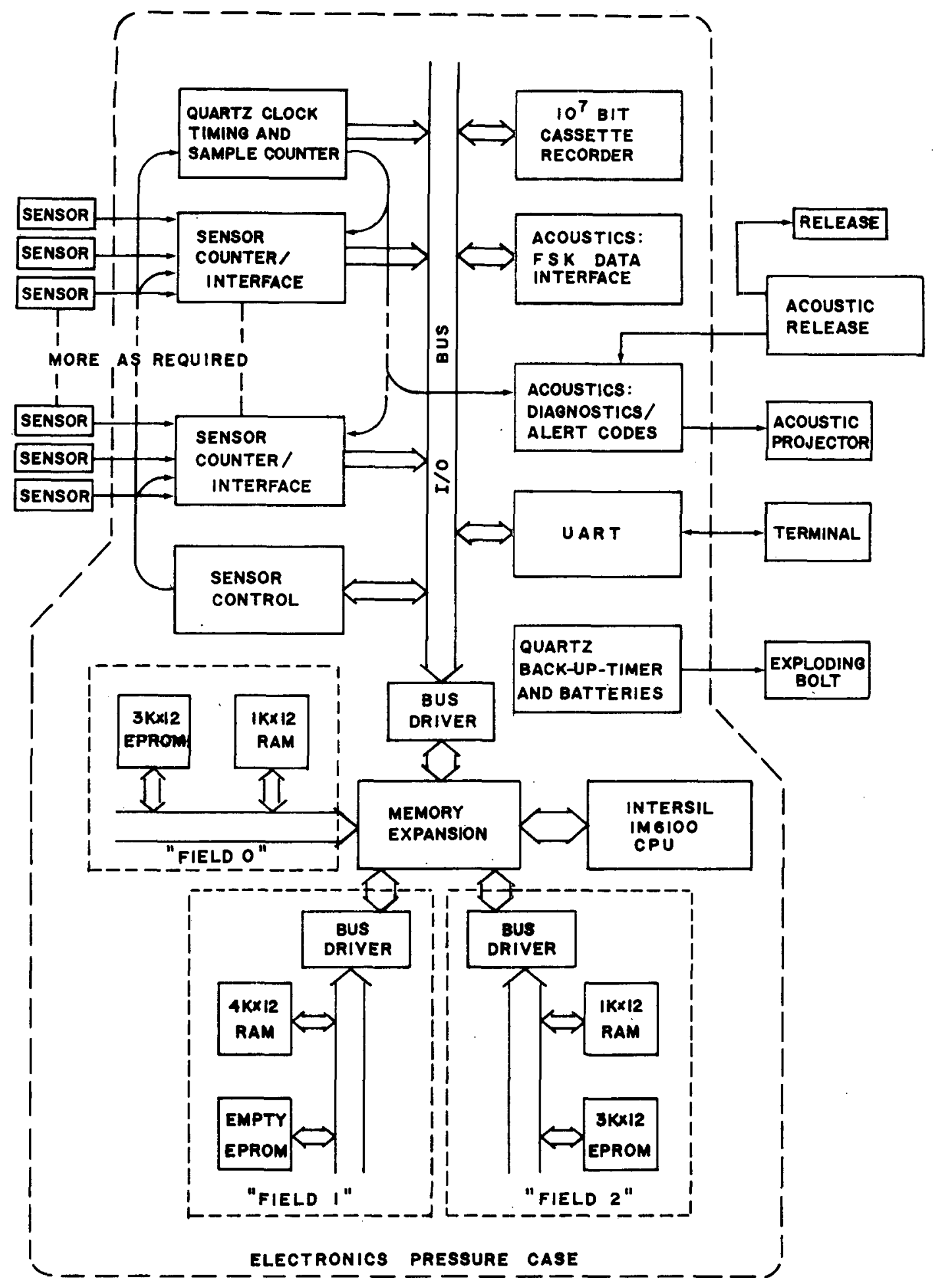

FIG. 1. Block diagram of the microprocessor-controlled recording system.

minute interval by standard frequency counting techniques, and is routinely recorded on cassette tape every 15 minutes independent of the conditional sampling scheme. This data set contains information on frequencies up to 2 cycles per hour (cph) and is more than adequate for tides and lower frequency variations. 
The variance of the 60 -sample set is also calculated and stored in RAM. This information is recorded as a low-frequency sample record which consists of an instrument identification word, sample number, the 15-minute sums and variances for each sensor.

The sixty $15 \mathrm{~s}$ samples of "high-frequency" data stored in the temporary memory contain information on frequencies between 4 and $120 \mathrm{cph}$. The conditional sampling algorithm which is discussed in more detail later is applied to these data and makes the decision whether or not to store the "high-frequency" data. If so, the 60 most recent samples of 15 -second data stored in RAM are written to the cassette tape. The instrument is constrained to record only a fixed number of events which will fill up the second half of the tape.

The entire electronics system shown in Fig. 1 is contained in an aluminum pressure housing one meter long and six inches in diameter. A second pressure housing holds up to 3 kilowatt hours of lithium batteries required to power the density array sensors for up to four months. A third pressure housing contains the batteries for the pressure sensors, the microprocessor electronics, and the tape recorder. These three pressure housings are mounted on an aluminum frame which supports the system components (see Fig. 2). The electronics and battery cases are mounted near the base of the frame. The $600 \mathrm{~kg}$ of buoyancy, acoustic transponder, current sensor, flashing light, and radio are positioned in the upper part of the frame. The package is held securely on the sea floor by a $2500 \mathrm{~kg}$ anchor. The density array is held taut above the bottom instrument by a subsurface float. After it is released from the bottom, by either a Sonatech acoustic release or timed exploding bolt, the instrument free floats to the surface for recovery. The reader is referred to a more detailed discussion of this instrumentation presented by Irish et al. (1983).

\section{Conditional sampling scheme}

The term "conditional sampling" is used here to refer to the following set of steps: preprocessing of the data, error detection, temporary storage of past data, event detection and final data storage. Preprocessing refers to any modification of the signal before it is stored or processed. Most instruments do some preprocessing of the data when it is filtered by a sensor or as it is digitized. In addition, other low pass or averaging filters are applied, and sometimes such derived quantities as salinity or Reynolds stress are calculated.

Error detection and noise reduction measures must be taken in order to deal with spurious values which may be encountered when the equipment is being launched or recovered, or at other times due to sensor or cable failure. Regardless of the cause, it is desirable to eliminate these spurious data before any conditional sampling calculations are performed. To detect spu- rious data, a simple error detector based on a firstdifference approach is employed. Geophysical signals are generally known well enough so that a definition of spurious first differences can be specified.

Past data storage is required so that the observations from the early stages of an event can be stored permanently. In our instrument the past 60 samples of temperature, pressure, conductivity, and current speed are temporarily stored in RAM and stored permanently only if an event is detected.

The simplest form of event detection initiates data storage when the signal exceeds a certain preset value. A higher order detection scheme initiates data storage when a pre-selected variance or energy level (rather than the level of the signal itself) is exceeded. Our conditional sampling scheme uses the energy in a selected frequency band (referred to as the "event window") for initiating high-frequency data storage. This process is discussed in detail in the following five sections.

\section{a. Preprocessing}

Before the actual conditional sampling algorithm is applied, the incoming data is processed in several ways. For example, the averaging done by counting the sensor frequency over the sample interval is an effective low pass filter which is usually adequate to suppress highfrequency fluctuations and reduce aliasing. The equivalent filter response of the averaging process, $H_{e}(f)$, is

$$
H_{e}(f)=\frac{\sin (\pi f \Delta)}{\pi f \Delta}
$$

where $f$ is the frequency and $\Delta$ the high-frequency sample interval. At the Nyquist frequency $(1 / 2 \Delta)$ the filter is down $4 \mathrm{~dB}$, and the envelope of the sidelobes decreases as $f^{-2}$. Further preprocessing includes computing the variance of the past 60 samples and salinity. In our application, salinity is calculated in situ from measured temperature and conductivity and a linearized equation of state before being processed by the conditional sampling algorithm. The salinity estimates are used only to detect the presence of special salinity or density related events. It is only the unprocessed temperature and conductivity data that are subsequently recorded if an event is detected. These preprocessed data are then subjected to the following elements of the conditional sampling scheme.

\section{b. First-differencing and error detection}

The first step of the conditional sampling scheme is the first-differencing of the raw data. Since most geophysical spectra decrease with increasing frequency 


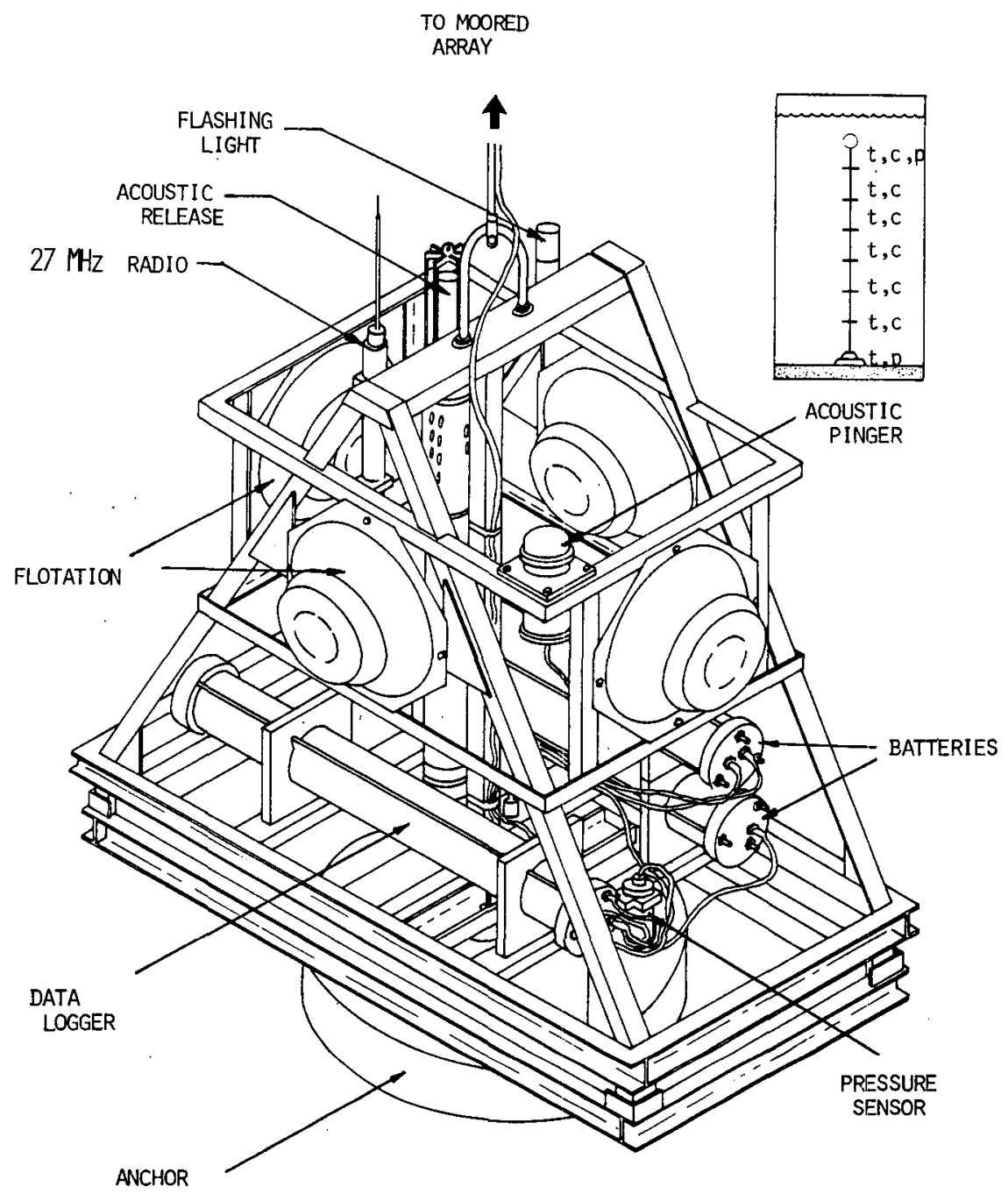

FIG. 2. UNH bottom-mounted instrument.

(red spectra), we employ a first-difference filter to suppress low frequency variation and pre-whiten the data.

To screen the data for errors, each first difference is compared with a preset level $\mathbf{B}$, which is larger than typical first differences expected from geophysical signals and yet small enough to eliminate spikes associated with instrument malfunction. In our application, a value of $B$ was chosen which is five times the maximum expected first difference. Specifically, the first difference record $p^{\prime}$ for a signal $p$ is computed according to

$$
p^{\prime}(t)=\left\{\begin{array}{l}
p(t)-p(t-1) \text { if } p^{\prime}(t) \leqslant B \\
p^{\prime}(t-1) \text { if } p^{\prime}(t)>B
\end{array}\right.
$$

The response of this first-differencing filter is

$$
p^{\prime}(t)=\int p(t-\tau) w(\tau) d \tau
$$

where $w(\tau)$ is the set of convolution weights

$$
w(\tau)=\delta(\tau)-\delta(\tau-\Delta)
$$

in which $\delta$ is the Kronecker delta and $\Delta$ is the 15second sample period. By the convolution theorem, the spectrum of a first-differenced record is the product of the record spectrum and the convolution weight spectrum, which is found by Fourier transforming the weights according to

$$
W(f)=\int_{-\infty}^{\infty} w(\tau) e^{-2 \pi i f \tau} d \tau=1-e^{2 \pi i f \Delta} .
$$

The spectral response shown in Fig. 3 is then

$$
W W^{*}=\left(1-e^{2 \pi i \Delta \Delta}\right)\left(1-e^{-2 \pi i f \Delta}\right)=4 \sin ^{2}(\pi f \Delta),
$$

where the asterisk denotes the complex conjugate. The phase lag of the filter is given by 


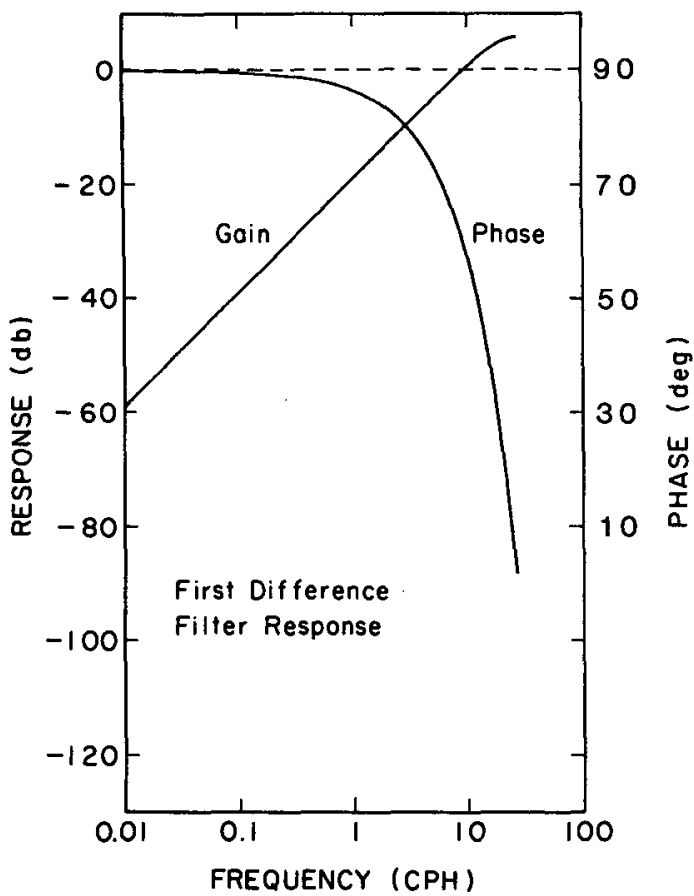

FiG. 3. The amplitude response (in $\mathrm{db}$ ) and phase response (in deg) of the first-differencing filter for discrete samples taken with a 64-second sample interval.

$$
\phi=\tan ^{-1}\left[\frac{\sin 2 \pi f \Delta}{1-\cos 2 \pi f \Delta}\right]=\left[\frac{\pi}{2}-\pi f \Delta\right]
$$

and is also shown in Fig. 3. The significant phase changes do not affect our results since only the signal magnitudes are used for event-detection. Anticipating tide filtering, the first differences are stored in a "press down" 60 -sample stack so the newest value is always available first.

\section{c. Anti-tide filter}

The dominant signal in oceanic records (especially pressure) is due to tidal oscillations. In order to select the higher frequency signal of interest, the tidal effects are removed with a digital filter applied to the firstdifferenced data. This filter is constructed from the weighted sum

$$
\begin{aligned}
p^{\prime}(t)+\alpha_{1} p^{\prime}\left(t-\Delta^{\prime}\right)+\beta_{1} p^{\prime}( & \left.t-2 \Delta^{\prime}\right) \\
= & \int p^{\prime}(t-\tau) W_{1}(\tau) d \tau,
\end{aligned}
$$

where $W_{1}(\tau)=1+\alpha_{1} \delta\left(\tau-\Delta^{\prime}\right)+\beta_{1} \delta\left(\tau-2 \Delta^{\prime}\right)$. The $W_{1}$ and $\Delta^{\prime}$ are not related to $W$ and $\Delta$ above, and $\Delta^{\prime}$ is an arbitrary lag chosen with $\alpha_{1}$ and $\beta_{1}$ to remove the diurnal tides. Similarly $W_{2}, \alpha_{2}$ and $\beta_{2}$ are chosen to remove the semidiurnal tides. By the convolution theorem, the response of the combined filters is

$$
\begin{aligned}
W(f)= & W_{1}(f) W_{2}(f) \\
= & \left(1+\alpha_{1} Z^{-1}+\beta_{1} Z^{-2}\right) \\
& \quad \times\left(1+\alpha_{2} Z^{-1}+\beta_{2} Z^{-2}\right),
\end{aligned}
$$

where $Z \equiv \exp \left(2 \pi i f \Delta^{\prime}\right)$. Multiplying and collecting terms leads to

$$
\begin{array}{r}
W(f)=1+\left(\alpha_{1}+\alpha_{2}\right) Z^{-1}+\left(\alpha_{1} \alpha_{2}+\beta_{1}+\beta_{2}\right) Z^{-2} \\
+\left(\alpha_{1} \beta_{2}+\alpha_{2} \beta_{1}\right) Z^{-3}+\beta_{1} \beta_{2} Z^{-4}
\end{array}
$$

which, when transformed back into the time domain, becomes

$$
\begin{aligned}
& w(\tau)=\delta(\tau)+\left(\alpha_{1}+\alpha_{2}\right) \delta\left(\tau-\Delta^{\prime}\right) \\
& \quad+\left(\alpha_{1} \alpha_{2}+\beta_{1}+\beta_{2}\right) \delta\left(\tau-2 \Delta^{\prime}\right) \\
& \quad+\left(\alpha_{1} \beta_{2}+\alpha_{2} \beta_{1}\right) \delta\left(\tau-3 \Delta^{\prime}\right)+\beta_{1} \beta_{2} \delta\left(\tau-4 \Delta^{\prime}\right) .
\end{aligned}
$$

A lag interval $\Delta^{\prime}$ of 15 samples is chosen so that the combined filter requires inputs from the previous $4 \Delta^{\prime}$ or 60 samples ( 15 minutes in this application). These inputs are obtained from the first-differenced "press down" stack.

The weights appropriate for the diurnal filter are determined from the spectral response

$$
\begin{aligned}
W_{1}(f) W_{1}(f)^{*}= & 1+\alpha_{1}{ }^{2}+\beta_{1}{ }^{2}+2 \alpha_{1}\left(1+\beta_{1}\right) \\
& \times \cos \left(2 \pi f \Delta^{\prime}\right)+2 \beta_{1} \cos \left(4 \pi f \Delta^{\prime}\right),
\end{aligned}
$$

where $\Delta^{\prime}=15$ samples ( 3.75 minutes). The diurnal tidal energy is concentrated in the two tidal lines $\mathrm{O}_{1}$ and $K_{1}$ with frequencies of $0.03861637 \mathrm{cph}$ and $0.04178074 \mathrm{cph}$, respectively. Their mean frequency is about that of the $\mathrm{M}_{1}$ tidal frequency, $f_{1}=0.040255$ $\mathrm{cph}$, which we take as a representative diurnal tidal frequency. Arbitrarily setting $\beta_{1}$ equal to 1.0 permits the computation of $\alpha_{1}$ in accordance with

$$
\alpha_{1}=-2 \cos \left(2 \pi f_{1} \Delta^{\prime}\right)=-1.9997501 .
$$

Thus, the spectral response of the diurnal filter,

$$
W_{1} W_{1}^{*}=16 \sin ^{2}\left[\pi\left(f+f_{1}\right) \Delta^{\prime}\right] \sin ^{2}\left[\pi\left(f-f_{1}\right) \Delta^{\prime}\right],
$$

has a notch at $f=f_{1}$.

Similarly, the semidiurnal tides have their principal energy at the $\mathrm{M}_{2}$ and $\mathrm{S}_{2}$ lines, with frequencies of $0.0805114 \mathrm{cph}$ and $0.0833333 \mathrm{cph}$, respectively. Since most of the energy is concentrated in the $\mathbf{M}_{2}$ line, $f_{2}$ is set equal to $0.08051 \mathrm{cph}$. Choosing $\beta_{2}=1.0$ and $\alpha_{2}$ $=-2 \cos \left(2 \pi f_{2} \Delta^{\prime}\right)=-1.9990005$ leads to the corresponding semidiurnal response.

The combined filter weights are

$$
\begin{aligned}
& w(0 \Delta)=\alpha_{0} \quad=1.0 \\
& w(15 \Delta)=\alpha_{1}+\alpha_{2} \quad=-3.9987506 \\
& \left.w(30 \Delta)=\alpha_{1} \alpha_{2}+\beta_{1}+\beta_{2}=5.9975015\right\} \text {. } \\
& w(45 \Delta)=\alpha_{1} \beta_{2}+\alpha_{2} \beta_{1}=-3.9987506 \\
& w(60 \Delta)=\beta_{1} \beta_{2} \quad=1.0
\end{aligned}
$$




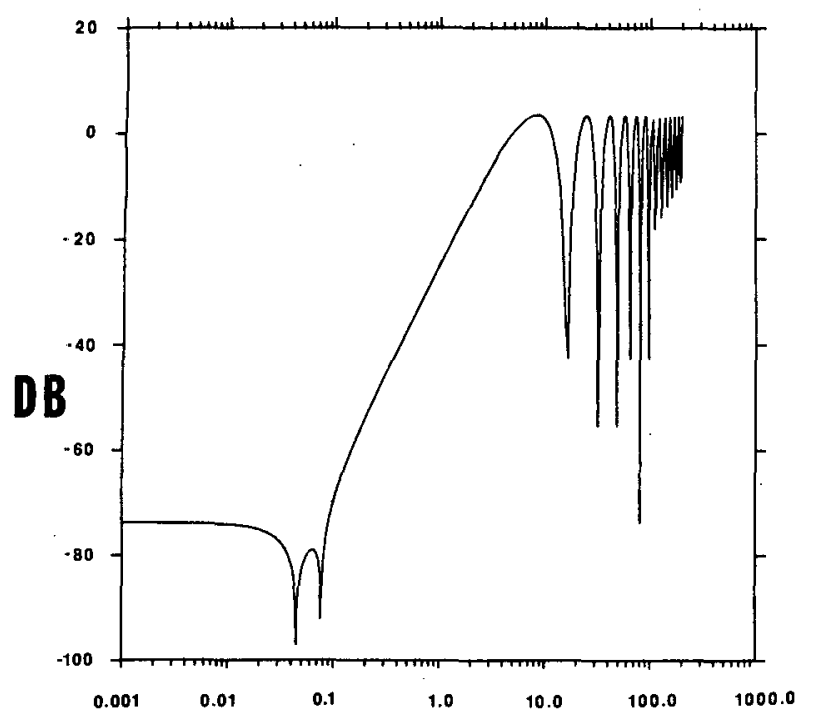

CPH

FIG. 4. The response of the composite "anti-tide" filter. The notches at high frequency are the result of the sum and differences of the diurnal and semidiurnal notch frequencies. The filter is normalized so that, on the average, the high-frequency energy is not attenuated.

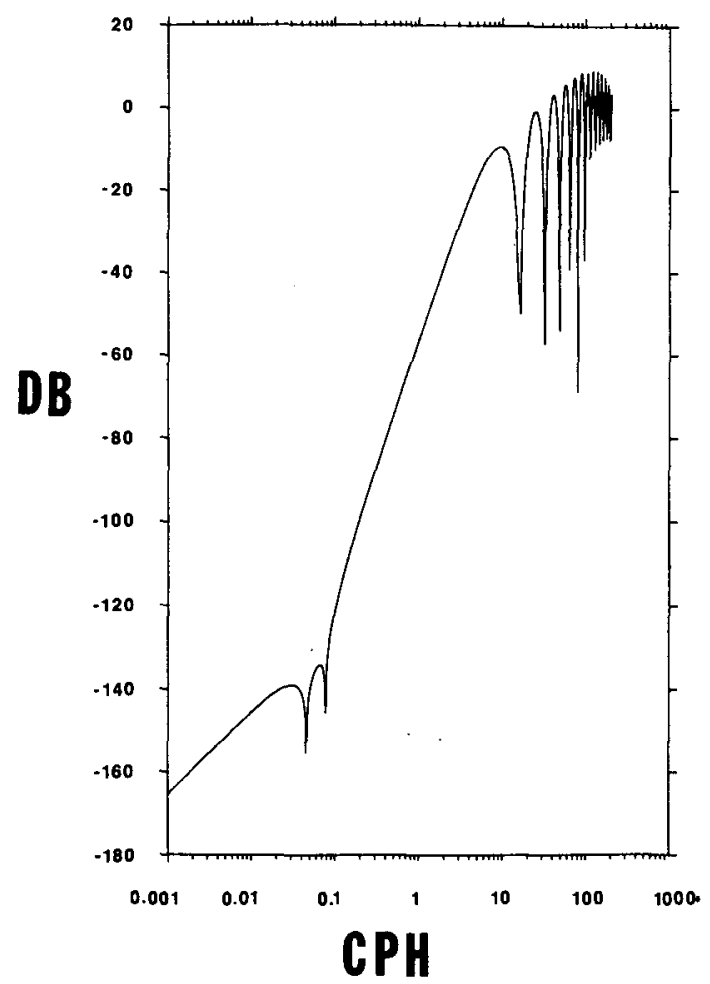

FiG. 5. The "event window" produced by the combined response of the averaging, first-difference, and "anti-tide" filter. Since the signal is digitized to discrete sample intervals, the data window is displayed out to the Nyquist frequency, above which it cuts off rapidly due to the averaging filter.
The spectral response of the composite "anti-tide" filter is shown in Fig. 4.

At this stage of data processing, the signal has been altered by the combined effects of the three filtering operations (averaging, first-differencing, and anti-tide filtering) described thus far. The product of these filters, which is shown in Fig. 5, defines the low-frequency end of the effective bandpass filtering operation which we call the "event window". Its effect is to select the high-frequency energy associated with the "events" of interest from a background of much more energetic lower frequency energy.

The usefulness of the event window is shown by an example of its application to some measurements on the New England shelf. The spectrum of the bottom pressure record in Fig. 6 shows the typically "red" background spectrum upon which the dominant tidal energy is superimposed. The effect of the event win-

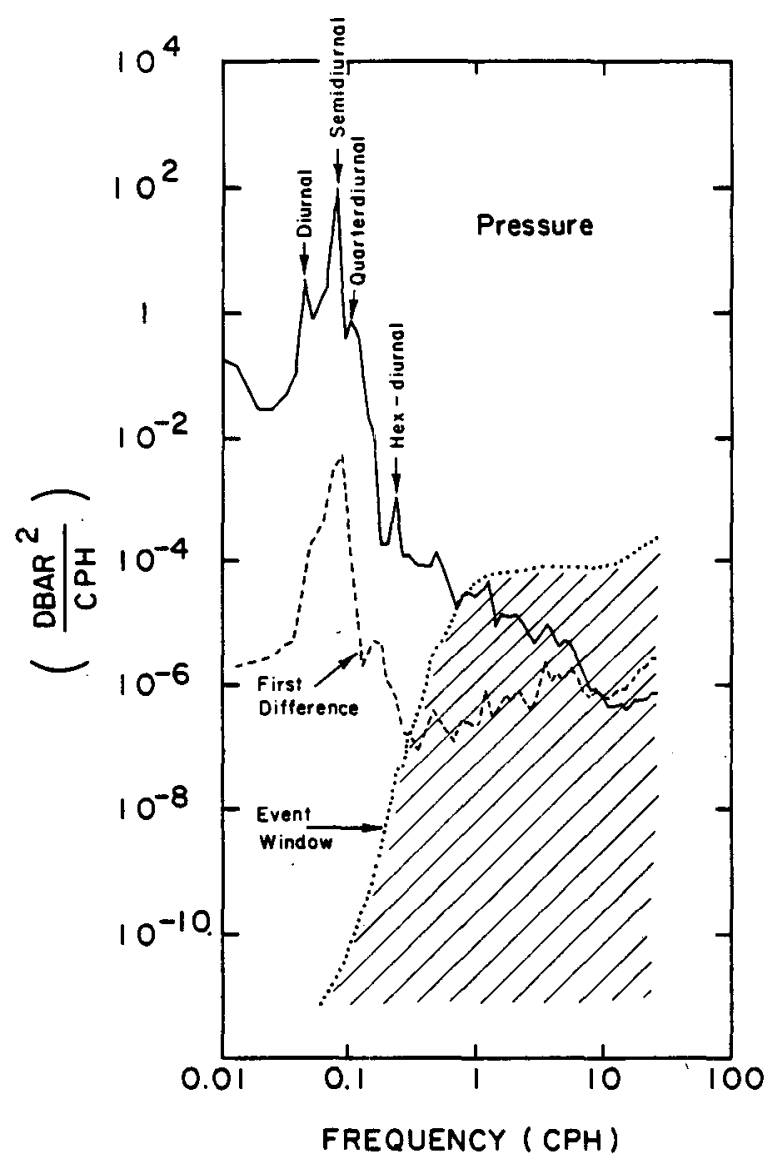

FiG. 6. A pressure spectrum from the New England continental shelf showing the typically dominant tidal peaks superimposed on a "red" spectrum. First-differencing suppresses the low-frequency energy, but the tides still dominate the spectrum. The "anti-tide" filter effectively removes this low-frequency energy leaving the energy in the "event window." The dotted line is the upper boundary or envelope enclosing the energy passed through the data window shown in Fig. 5. The shaded region represents the energy used for event detection. 
dowing process is to pass only that part of the pressure energy enclosed in the shaded region in Fig. 6. For this particular application, $\Delta$ was 64 seconds, so the effective bandwidth of the event window covers frequencies from 0.5 to $28 \mathrm{cph}$.

\section{d. Intensity calculation}

The actual event detection is triggered when the intensity of the event-windowed signal exceeds a certain "critical" level. The intensity $\tilde{p}_{t}$ is defined as a weighted sum of past and present values and is computed from the event windowed signal $p_{t}^{\prime \prime}$ according to

$$
\tilde{p}_{t}=\gamma \tilde{p}_{t-1}+(1-\gamma)\left|p_{t}^{\prime \prime}\right|,
$$

where $\gamma=0.9835$ is an exponential fading with a time constant equal to 60 samples (15 minutes).

The behavior of the intensity calculation can be illustrated with two simulations. In the first, shown in Fig. 7 , the original record, $p_{t}$ has a step change of $\Delta_{p}$ $=100$ counts at $t_{0}$. The output of the first-difference filter $p_{t}^{\prime}$ consists of a spike with an amplitude of 100 at $t_{0}$. The output from the anti-tide filter, $p_{\imath}^{\prime \prime}$, is a series of five spikes with amplitudes of the weights $W$ times $100(100,-400,600,-400,100)$ at lags of $1,15,30$, 45 , and 60 samples, respectively. The intensity $\tilde{p}_{t}$ reaches its maximum value $\tilde{p}_{\max }=0.1503 \Delta p, 11.25$

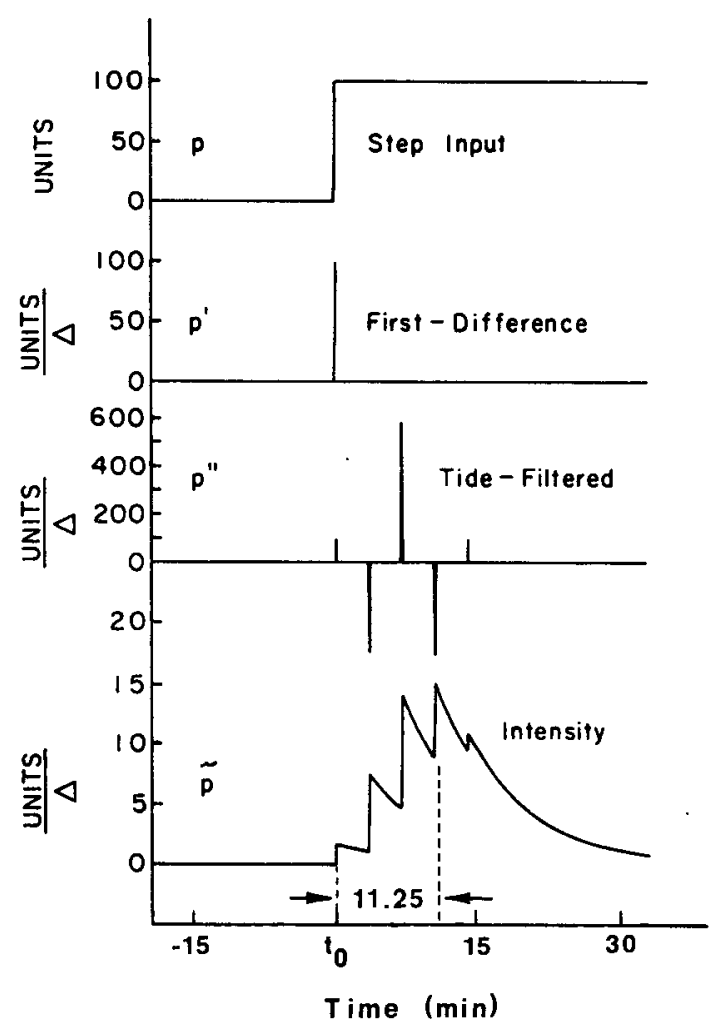

FIG. 7. The response of the conditional sampling algorithm to a step change in input.

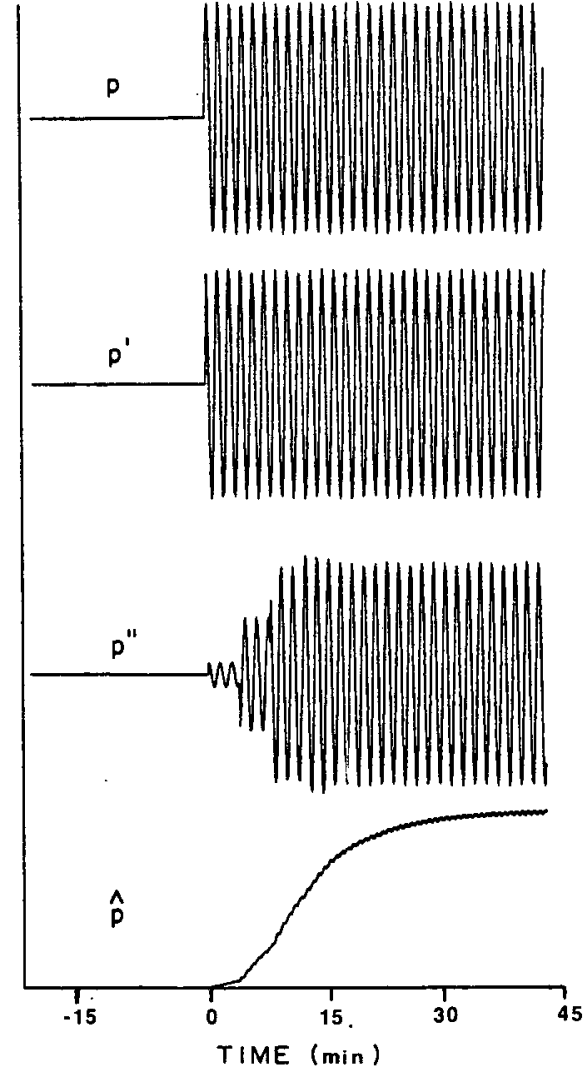

FIG. 8. The response of the conditional sampling algorithm to the onset of a sine wave.

minutes after the time of the step in the original series. In the second simulation, shown in Fig. 8, the outputs of the different components of the conditional sampling scheme due to the sudden imposition of a sinusoidal signal are illustrated. In this case, the intensity builds up to its asymptotic value after about 30 minutes, which is a measure of the characteristic time required for the intensity output to equilibrate to the sea floor environment at the beginning of an experiment.

\section{e. Adaptive critical and the event identifier}

An event is identified when the intensity $\tilde{p}_{t}$ for a sensor exceeds the appropriate "critical" value $D$. An intelligent choice of $D$ depends on some knowledge of the "background" environmental energy levels to be encountered. If the value chosen for $D$ were too large, no events would be recorded; while choosing too small a value for $D$ would cause uninteresting data to be recorded continually, thereby filling the storage capacity prematurely.

The difficulty in intelligently choosing critical intensity values for new environments became clear after the first test of the conditional sampling scheme in the Gulf of Maine in 1979. During this test, high-frequency data were recorded continuously because excessively 
small constant critical intensity values were selected. This result demonstrated the need for an automatic procedure for adapting the critical values to the statistics of an unfamiliar environment (Irish et al., 1981a).

We have chosen an adaptive scheme in which a time-variable critical intensity value is determined from the statistics of the intensity itself. This so-called adaptive critical intensity $D_{a}$ is defined according to

$$
D_{a}=\bar{p}_{k}+2 \sigma_{k} \text {, }
$$

where the intensity mean $\bar{p}_{k}$ is defined as

$$
\bar{p}_{k}=\frac{1}{k} \sum_{i=1}^{k} \tilde{p}_{i}
$$

and the intensity standard deviation $\sigma_{k}$ is defined as

$$
\sigma_{k}^{2}=\frac{1}{k} \sum_{i=1}^{k}\left[\tilde{p}_{i}-\bar{p}_{k}\right]^{2}
$$

Both these statistics are based on the accumulation of results from the beginning of the experiment through the most recent sample number $k$ and of course become more stable as the experiment proceeds.

A simulation of the adaptive critical level scheme as applied to the high-frequency data from the Gulf of Maine is shown in Fig. 9, in which a recreation of the first-differenced, tide-filtered, and intensity time series is presented. An adaptive critical value computed from the above relation is shown in relation to the intensity output. If this scheme had been implemented at the time of these measurements, an event would have been declared around the time of the large intensity peak on 2 July 1979 . The overall results from those measurements show that this event was probably

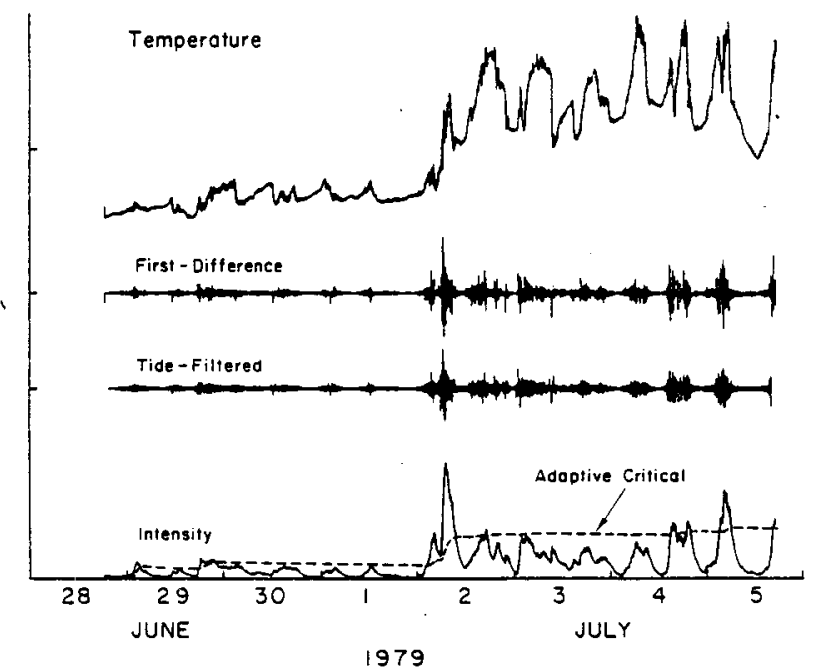

FIG. 9. A Gulf of Maine temperature time series with a simulation of the first-differencing and "anti-tide" filters, intensity and adaptive critical calculations. due to the high-frequency turbulent fluctuations associated with the intrusion of a warm water front.

Once the intensity for a particular parameter has exceeded its critical level, adaptive or otherwise, the following set of criteria must be satisfied before the data in the 60-sample buffer are recorded:

1) The intensity $\tilde{p}$ must be greater than the adaptive critical intensity $D_{a}$ since the last data storage, indicating an event has occurred.

2) The sensor must be functioning properly. That is, if there were too many spurious first differences indicating spurious data, the algorithm will then prevent that sensor from triggering a critical for one day.

3) A least 60 samples must have been taken since the last command to record event data.

4) The event must not be too long. That is, the number of criticals, which initiate data recording from the present event, must be fewer than the preset limit of events. Separate events are operationally defined by the absence of a "critical" for a fixed number of samples. Thus, marginal intensity data at the end of one event will be assigned to that event.

5) The number of events associated with a particular parameter must not be excessive. The number of criticals, which initiate storage of data, must be less than the number selected for a particular experiment.

If all these conditions are met, then the unprocessed high-frequency samples stored in memory for all sensors are recorded on cassette tape.

\section{Results and discussion}

The first use of our conditional sampling scheme with the adaptive critical feature was as part of a test deployment of a temperature/conductivity array in Massachusetts Bay in 1980. For this deployment, a vertical array of six pairs of temperature and conductivity sensors was added to the bottom-mounted instrument on which temperature, pressure and speed sensors were mounted. The temperature and conductivity on the array and the bottom pressure were conditionally sampled. The low-frequency records (20 minute samples) from the six temperature sensors are presented at the top of Fig. 10. Of the several events which were detected, one at the $42 \mathrm{db}$ depth is highlighted. The high-frequency or conditionally sampled (20-second samples) temperatures from this event are presented on an expanded time scale at the bottom of Fig. 10. (Note that the temperature scales are the same.) The high-frequency oscillations at the local BruntVäisälä frequency $(6 \mathrm{cph})$ can be seen clearly during the final third of the event (Irish et al., 1981b).

There were obvious salinity features which were inadequately resolved with the routine low-frequency sampling during the Massachusetts Bay 1980 deployment. Since a conductivity record is very similar to that of temperature, it does not provide additional 

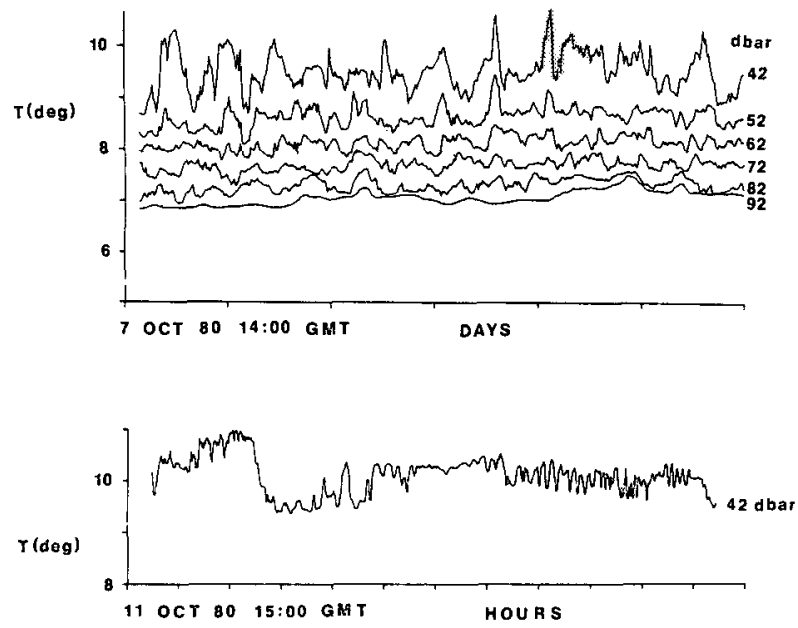

FiG. 10. (Top) plot of temperature time series, sampled at low frequency (20-minute intervals), from the Massachusetts Bay 1980 deployment. (Bottom) the high-frequency or conditionally sampled (20-second) temperature time series at $42 \mathrm{db}$, corresponding to the shaded region above, presented on an expanded time scale for the duration of the event.

information for detecting events, especially those related to salinity. As a result of these tests, the conditional sampling algorithm was modified to include a computation of an in situ salinity from the temperature and conductivity using a linearized equation of state. In subsequent work we have used the computed salinity series as well as temperature and pressure series for conditional sampling purposes.

The first scientific deployment of a conditional sampling temperature/conductivity array instrument occurred during the Coastal Ocean Dynamics Experiment (CODE), which was conducted on the northern $\mathrm{Cal}$ ifornia continental shelf during the summers of 1981 and 1982 (The CODE Group, 1983). During the three month depioyment in the summer of 1981,101 events were triggered by temperature, pressure and/or salinity. The high-frequency salinity time series at $123 \mathrm{~m}$ depth from events 39 and 71 are presented in Fig. 11. Event 71 was initiated solely by the salinity fluctuations shown, while event 39 was principally a temperature event higher up in the water column. The salinity variance of event 71 is an order of magnitude larger than that of event 39 . The critical intensity "flag" series are shown below each salinity series. This flag is high for a 15-minute duration if the adaptive critical was exceeded by the intensity any time during that 15 -minute record. As can be seen in Fig. 11, the significant salinity fluctuations can be detected in practice by the conditional sampling algorithm described here.

We have modified the adaptive critical scheme for repetitive deployments in the same region by setting an initial adaptive critical for the new experiment equal to the final critical from the preceding experiment. This initial critical is given a weight which is equivalent to the instrument having been in the water collecting statistics for one month. Thus, the dominant statistics determining $D_{a}$ during the start of the new deployment are those accumulated in the ast deployment. As the new experiment progresses, its statistics dominate the determination of the adaptive critical.

More complicated schemes for determining the adaptive critical would draw on a knowledge of both the length of the experiment and the remaining data storage space in adjusting the critical level so that the tape would be filled at the end of the experiment. In our work on this option, unresolved questions are: 1) at what point in the experiment modification of $D_{a}$ should begin, 2) how often $D_{a}$ should be reexamined and readjusted, and 3) what cbjective scheme should be used to determine the size of the adjustment. Studies of past data indicate that $D_{a}$ should be modified only after the first one-third to one-half of the experiment has elapsed, after which it should be reexamined every one-tenth of an experiment length and adjusted by one-third of a standard deviation.

Although we have presented a specific solution to the conditional sampling problem, it is obvious that various modifications can be made using parts of our scheme. Having worked with conditional sampling instruments in several different configurations, and considering the recent improvement in capacity of remote recorders, we are now able to refine our philosophy in the application of conditional sampling techniques to oceanic measurements. When an exploratory experiment is planned where the spatial and temporal scales of the dominant processes are not known, the use of a high-capacity recorder with maximum sam-

\section{SALINITY}
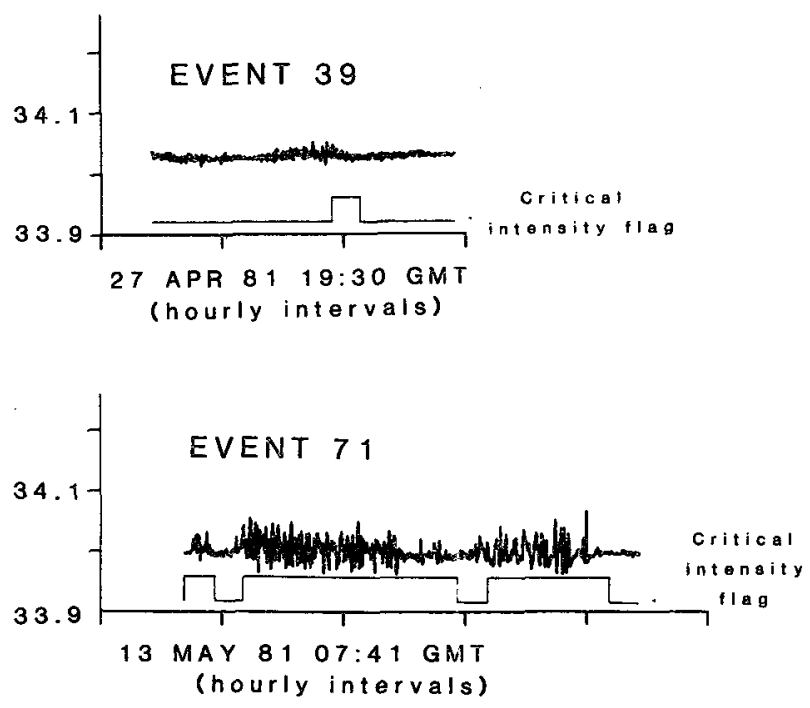

FIG. 11. CODE-1 salinity events 39 and 71 plotted with the critical intensity flag. This flag is high if an event was detected by that sensor during a $15 \mathrm{~min}$ period. The record shown is continuous when the flag is low because events were detected at other levels by temperature, salinity or pressure. 
pling speed will resolve the largest frequency band and allow exploratory studies to be made on the dominant processes involved. When the experiment is designed to study the behavior of a particular intermittent process with a rather well-defined behavior, then a conditional sampling scheme is best employed. Conditional sampling techniques are useful in: 1) deep ocean tide station studies of tsunamis, wherein a narrow frequency band can be selected and the algorithm discussed above applied, 2) studies of winter cooling and overturning wherein an event could be defined as a nearly uniform temperature at various depths along an array, and 3) studies of sediment erosion and transport wherein a specific relationship between velocity and suspended sediments can be defined. Questions concerning other intermittent processes like internal solitons, storm tides, and bottom water formation can also be addressed using the basic techniques presented here.

Acknowledgments. Many of the basic ideas behind this work evolved out of a collaborative effort started in the early 1970s at Scripps Institution of Oceanography under the direction of Walter Munk and Frank Snodgrass. The authors acknowledge the guidance provided by both of them. A prototype instrument was constructed by the Marine Systems Engineering Laboratory at UNH with funding by the Leslie S. and Iola M. Hubbard Marine Program Fund. Mark Woodbury, Mitchell Condylis and Alvin Bugbee are ac- knowledged for the efforts in connection with the development of both hardware and software components of this instrumentation. The initial software development, instrument deployment, and analysis was funded by the National Science Foundation Grant OCE-7826229 and the Office of Naval Research Contract N00014-80-C-0351. The work during CODE was funded by NSF under Grant OCE-8014940.

\section{REFERENCES}

Irish, J. D., W. S. Brown and M. P. Woodbury, 1981a: Conditional sampling of oceanic temperature and pressure. Ocean Process Analysis Laboratory Tech. Rep. No. 8101, University of New Hampshire, $46 \mathrm{pp}$.

_- - - and - $198 \mathrm{lb}$ : Conditional sampling of oceanic variability with microprocessor-controlled instrumentation. Proc. Oceans '81, IEEE, Boston, 484-488.

-, M. P. Woodbury and E. J. LaCoursiere, 1983: A bottommounted, microprocessor-controlled instrument system. Ocean Process Analysis Laboratory, Tech. Rep. OPAL-83-1, University of New Hampshire, $43 \mathrm{pp}$.

Pederson, A. M., 1969: An accurate low-cost temperature sensor. Trans. Marine Temperature Measurements Symp., Miami, Mar. Tech. Soc., 135-153.

- - and M. C. Gregg, 1979: Development of a small in-situ conductivity instrument. IEEE J. Oceanic Eng., OE4, 69-75.

Snodgrass, F. E., 1968: Deep sea instrument capsule. Science, 162, 78-87.

The CODE Group, 1982: A preliminary description of the CODE1 field program. Tech. Rep. No. 82-51, Woods Hole Oceanographic Institution, $47 \mathrm{pp}$.

Wearn, R. B. Jr., and N. G. Larson, 1982: Measurements of the sensitivities and drift of digiquartz pressure sensors. Deep-Sea Res., 29, 111-134. 